\title{
Evaluation of Multiple Reaction Monitoring Cubed for the Analysis of Tachykinin Related Peptides in Rat Spinal Cord using a Hybrid Triple Quadrupole-Linear Ion Trap Mass Spectrometer
}

Floriane Pailleux and Francis Beaudry*

Groupe de Recherche en Pharmacologie Animal du Québec (GREPAQ), Département de biomédecine vétérinaire, Faculté de médecine vétérinaire, Université de Montréal, St-Hyacinthe, Québec, Canada

"Corresponding author:

Francis Beaudry, Ph.D.

Assistant Professor in Analytical Pharmacology

Département de Biomédecine Vétérinaire

Faculté de Médecine Vétérinaire

Université de Montréal

3200 Sicotte

C.P. 5000

Saint-Hyacinthe, QC

Canada J2S 7C6

Email: francis.beaudry@umontreal.ca

Tel (514) 343-6111 ext. 8647

Keywords: Tachykinins, Neuropeptides, Mass spectrometry, Multiple Reaction Monitoring, Biomarkers 


\section{Abstract}

Targeted peptide methods generally use HPLC-MS/MRM approaches. Although dependent on the instrumental resolution, interferences may occur while performing analysis of complex biological matrices. HPLC-MS/MRM ${ }^{3}$ is a technique, which provides a significantly better selectivity, compared with HPLC-MS/MRM assay. HPLC-MS/MRM ${ }^{3}$ allows the detection and quantitation by enriching standard MRM with secondary product ions that are generated within the linear ion trap. Substance P (SP) and neurokinin A (NKA) are tachykinin peptides playing a central role in pain transmission. The objective of this study was to verify whether HPLC$\mathrm{MS} / \mathrm{MRM}^{3}$ could provide significant advantages over a more traditional HPLC-MS/MRM assay for the quantification of SP and NKA in rat spinal cord. The results suggest that reconstructed $\mathrm{MRM}^{3}$ chromatograms display significant improvements with the nearly complete elimination of interfering peaks but the sensitivity (i.e. signal-to-noise ratio) was severely reduced. The precision (\%CV) observed was between 3.5\% - 24.1\% using HPLC-MS/MRM and in the range of $4.3 \%-\mathbf{1 3 . 1 \%}$ with HPLC-MS/MRM ${ }^{3}$, for SP and NKA. The observed accuracy was within $10 \%$ of the theoretical concentrations tested. HPLC-MS/MRM ${ }^{3}$ may improve the assay sensitivity to detect difference between samples by reducing significantly the potential of interferences and therefore reduce instrumental errors.

\section{Abbreviations:}

MRM: Multiple Reaction Monitoring; $\mathrm{MRM}^{3}$ : Multiple Reaction Monitoring Cubed HPLC: High Performance Liquid Chromatography; MS: Mass Spectrometry; ESI: Electrospray ionization; SP: Substance P; NKA: Neurokinin A; TFA: trifluoroacetic acid 


\section{Introduction}

Neuropeptides play a central role in pain transmission and their quantification provides valuable insight on the molecular events associated with the pathophysiology. Moreover, neuropeptides can be used as biomarkers to support the development of new analgesic drugs and assist the decision making process in non-clinical drug development [1-3]. Mass spectrometry (MS) has a significant role in bioanalytical chemistry workflow. Adequate method selectivity, precision and accuracy are essential and both HPLC-MS/MRM and HPLC-MS/MRM ${ }^{3}$ represent interesting options when performing analysis on a hybrid triple quadrupole-linear ion trap mass spectrometer (QqLIT). The vast majority of targeted peptide assays use HPLC-MS/MRM however interferences often occurs while performing analysis of complex biological matrices. HPLC$\mathrm{MS} / \mathrm{MRM}^{3}$ is a technique which enables a better selectivity compared with a more conventional HPLC-MS/MRM assay and it can be performed on a hybrid QqLIT MS. This analytical approach allows the detection and quantitation by enriching standard MRM with secondary product ions that are generated within the linear ion trap. This strategy is particularly suitable when high background and/or interferences are observed in complex biological matrices (e.g. organs, plasma) and consequently standard MRM methods do not provide adequate selectivity thus affecting the assays figure of merits. Recently, a HPLC-MS/MRM ${ }^{3}$ analytical method was proposed for routine analysis of low-abundance putative protein biomarkers using surrogate tryptic peptides [4]. The authors demonstrated that a technique using in silico reconstructed $\mathrm{MRM}^{3}$ chromatograms using specific secondary ions produced from a trapped primary product ion, provided enhancements of the selectivity and figures of merits of the assay allowing the analysis of targeted protein biomarkers at trace concentrations in human serum. Thus, our objective was to verify if this analytical strategy could provide significant advantages over a more 
traditional HPLC-MS/MRM assay for the quantification of specific tachykinin peptides in rat spinal cord.

Substance P (SP) and neurokinin A (NKA) are tachykinin peptides playing a central role in pain transmission [5]. SP and NKA are both pronociceptive peptides and agonists of the receptor neurokinin-1 (NK1) present in the lamina I of the spinal cord [6-7]. However, NKA binds preferentially to the receptor neurokinin-2 (NK2) expressed at the lower level of the spinal cord. The interaction frequency between SP, NKA and their postsynaptic receptors strongly correlates with the intensity and duration of pain [5]. Moreover, there is a close link between the upregulation of SP and NKA with an increased release of excitatory amino acids in the intersynaptic space [8]. SP and NKA are essential elements in pain transmission and secondary hyperalgesia. SP and NKA are both important biomarkers used for preclinical pain pharmacology assessments. Consequently, the development of a selective, sensitive, precise and accurate analytical method is essential to test our hypotheses linking SP and NKA with pain behaviors.

\section{Materials and Methods}

\subsection{Chemicals}

Substance P and neurokinin A were purchased from Phoenix Pharmaceutical (Belmont, CA, USA). Deuterium labeled analog peptides were synthesized and used as internal standards (CanPeptide, Inc., Pointe-Claire, QC, Canada). Acetonitrile was purchased from Fisher Scientific (NJ, USA). Trifluoroacetic acid (TFA) and formic acid (FA) were purchased from J.T. Baker (Phillipsburg, NJ, USA). Standard solutions were prepared using a $0.25 \%$ TFA aqueous solution. 


\subsection{Sample preparation}

Deuterated peptides were specifically labeled on glycine and leucine residues (SP:

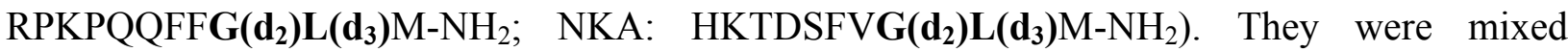
together and diluted in a $0.25 \%$ TFA aqueous solution at a final concentration of $500 \mathrm{ng} / \mathrm{mL}$. Rat spinal cord tissues were spiked with SP and NKA at concentrations of 50, 100 and $250 \mathrm{pg} / \mathrm{mg}$ of tissues and analyzed. The calculation of the method accuracy took into account the endogenous basal concentrations and standards were prepared in an alternative solution. The most suitable solution was to use $0.25 \%$ TFA solution since this is the solution used for tissue homogenization. Standards were prepared at concentrations ranging from 20 to $500 \mathrm{ng} / \mathrm{mL}$. To calculate the accuracy, the endogenous level needed to be subtracted from the observed concentration of fortified rat spinal cord tissues $(\% \mathrm{NOM}=([$ measured concentration $]-[$ measured endogenous concentration $] /[$ [spiked concentration $] \times 100$ ). Furthermore, tissue processing is an important step to preserve the sample integrity and adequately stabilize endogenous peptides. Further details can be found a previously published article [9]. The animals were euthanized with an overdose of isoflurane followed by a transection of the cervical spine. A flush of saline was performed within the spinal canal to collect the spinal cord lumbar enlargement. Tissue samples were snap-frozen in cold hexane $\left(\approx-60{ }^{\circ} \mathrm{C}\right)$ and stored immediately at $-80^{\circ} \mathrm{C}$ pending analyses. The rat tissues were weighed accurately and homogenized using a Tissue Tearor following the addition of $0.25 \%$ TFA solution at a ratio of $1: 5(\mathrm{w}: \mathrm{v})$. Normally, the spinal cord lumbar enlargement weighs between 150-200 mg for animals weighing 250-350 g (7 to 10 weeks of age). The samples were sonicated for $20 \mathrm{~min}$ and $150 \mu \mathrm{L}$ of the homogenate were mixed with $150 \mu \mathrm{L}$ of acetonitrile to precipitate high molecular weight proteins. The samples were vortexed and centrifuged at $12,000 \mathrm{~g}$ for $10 \mathrm{~min}$. Then $150 \mu \mathrm{L}$ of the supernatant were mixed with $150 \mu \mathrm{L}$ of 
the internal standard solution (deuterium labeled peptides). The samples were then transferred into an injection vial for analysis.

\subsection{Bioanalytical method}

The HPLC-MS/MRM and HPLC-MS/MRM ${ }^{3}$ analyses were performed using a Perkin Elmer Series 200 HPLC system (Shelton, CT, USA) coupled with an AB SCIEX API 2000 QTRAP $^{\circledR}$ hybrid triple quadrupole-linear ion trap mass spectrometer (Concord, Ontario, Canada). Ten $\boldsymbol{\mu} \mathbf{L}$ were injected onto a Thermo Biobasic C8 $100 \times 2.1 \mathrm{~mm}(5 \mu \mathrm{m})$ and peptides were separated using a gradient mobile phase. The initial mobile phase condition consisted of acetonitrile (A) and water (B) both fortified with $0.4 \%$ (v/v) of formic acid and was maintained at a ratio of 5:95 (A:B) from 0 to $1 \mathrm{~min}$. From 1 to 13 min a linear gradient was applied and a ratio of 60:40 (A:B) was maintained from 13 to $15 \mathrm{~min}$. At $15.1 \mathrm{~min}$, the mobile phase composition was reverted to 5:95 (A:B) and the column was allowed to equilibrate for $10 \mathrm{~min}$ for a total run time of $25 \mathrm{~min}$. The flow rate was fixed at $300 \mu \mathrm{L} / \mathrm{min}$ and SP and NKA eluted at 13.1 and 12.4 min respectively. The eluates were analyzed using an AB SCIEX API 2000 QTRAP mass spectrometer interface with pneumatic assisted electrospray ion source. The nitrogen gas 1 was set to 25 PSI, the nitrogen gas 2 was set to 40 PSI and the electrospray electrode was set to $5000 \mathrm{~V}$. The declustering potential (DP) was set to $30 \mathrm{~V}$. The mass spectrometer was operated in MRM or $\mathrm{MRM}^{3}$ mode using specific mass transitions with a collision energy set to $22 \mathrm{~V}$. The MRM transitions were acquired with a dwell time fixed to $200 \mathrm{~ms}$ for each mass transition. In $\mathrm{MRM}^{3}$ mode, ion trap fill time and excitation time was set to 200 and $25 \mathrm{~ms}$, respectively. In all $\mathrm{MRM}^{3}$ experiments, the scan rate was set to $\mathbf{4 0 0 0} \mathrm{amu} / \mathrm{s}$ and a $\mathrm{Q} 0$ trapping was used. Details on selected ions used for HPLC-MS/MRM and HPLC-MS/MRM³ analysis are reported in Table 1. Regression analyses were performed with PRISM (version 6.0c) GraphPad software (La Jolla, CA, USA) using nonlinear curve-fitting module with an estimation of the goodness of fit. The 
calibration lines were constructed from the light and heavy peptide isotopes peak-area ratios of targeted neuropeptides.

\section{Results and discussion}

Full-scan MS, $\mathrm{MS}^{2}, \mathrm{MS}^{3}$ spectra were recorded in positive mode to determine the most sensitive MRM or $\mathrm{MRM}^{3}$ transitions to be used for the quantification of SP and NKA. The full-scan electrospray mass spectrum showed the formation of characteristic $[\mathrm{M}+\mathrm{nH}]^{\mathrm{n+}}$ pseudo-molecular ions with the doubly charged species being the most abundant for both peptides as shown in Figure $1 \mathrm{~A}$ and $1 \mathrm{~B} . \mathrm{MS}^{2}$ mass spectra of $[\mathrm{M}+2 \mathrm{H}]^{2+}$ ions (Fig. $1 \mathrm{C}$ and $1 \mathrm{D}$ ) and $\mathrm{MS}^{3}$ mass spectra (Fig. 1E and 1F) are dominated by b-type fragment ions with some others (a, y and z), based on the Roepstorff and Fohlman nomenclature (Roepstorff and Fohlman, 1984). Suitable MRM and $\mathrm{MRM}^{3}$ transitions were thus defined from the selection of the two or three most abundant fragment ions for MRM and $\mathrm{MRM}^{3}$ respectively. An $\mathrm{MRM}^{3}$ ion chromatogram is reconstructed from selected specific fragment ions generated from the most abundant primary product ion trapped in the Q3 linear ion trap and then activated by resonant excitation [4]. To evaluate the improvement in selectivity using $\mathrm{MRM}^{3}$ compared to the conventional MRM, we compared chromatogram from the analyses of endogenous SP and NKA from rat spinal cord. The MRM chromatograms of the targeted mass transitions for both peptides revealed some interfering peaks as illustrated in Fig 2. Other transitions were tested but the sensitivity was seriously compromised and therefore impaired our ability to detect endogenous SP and NKA without performing further sample preparation steps. Interferences may significantly impact the assay precision, accuracy and robustness. As shown in Fig 2, reconstructed $\mathrm{MRM}^{3}$ chromatograms display significant improvements with the nearly complete elimination of interfering peaks. These $\mathrm{MRM}^{3}$ chromatograms were generated using the three most abundant secondary $\mathrm{MS}^{3}$ fragment ions and 
was judged to be the best compromise in order to preserve adequate sensitivity and improve selectivity. However, the sensitivity (i.e. signal-to-noise ratio) was severely reduced as illustrated in Fig.2 and could be an important limiting factor for the analysis of low abundant peptides. The measured endogenous levels for the tested rat spinal cords $(n=6)$ were $53.1( \pm 9.5)$ $\mathrm{pg} / \mathrm{mg}$ and 60.1 ( \pm 7.8$) \mathrm{pg} / \mathrm{mg}$ for SP and NKA respectively using HPLC-MS/MRM. The results were not statistically different using HPLC-MS/MRM ${ }^{3}$ with endogenous levels of $49.6( \pm 7.1)$ $\mathrm{pg} / \mathrm{mg}$ and $57.3( \pm 5.9) \mathrm{pg} / \mathrm{mg}$. The AB SCIEX API 2000 QTRAP instrument sensitivity in $\mathrm{MRM}^{3}$ was sufficient to analyze these two peptides in rat spinal cords but more sensitive instruments would be required to analyze these peptides in CSF or plasma since concentrations are significantly lower (i.e two order of magnitude). The precision and accuracy were assessed and compared between both HPLC-MS/MRM and HPLC-MS/MRM ${ }^{3}$ methods using spiked rat spinal cord tissue homogenates with SP and NKA at concentration of 50, 100 and 250 pg/mg. Each spiked sample was analyzed in six replicates including blanks along with standards prepared at concentrations ranging from 20 to $500 \mathrm{ng} / \mathrm{mL}$ to measure endogenous levels. In Table $\mathbf{2}$, the precision and accuracy data showed that both methods provided acceptable figures of merit with a marked better precision at the low concentrations in HPLC-MS/MRM ${ }^{3}$. As shown in Fig 2, interferences are present in HPLC-MS/MRM and it will inherently affect peak integration and consequently the precision of the method. Generally, analysis of neuropeptides are performed near the endogenous values and the added improvement in selectivity provided by HPLC$\mathrm{MS} / \mathrm{MRM}^{3}$ analysis may have a significant positive impact on the precision of the measured signal and consequently improve the sensitivity of the method to detect biologically significant differences between samples. 


\subsection{Conclusion}

The strict comparison between the statistics of the analysis of SP and NKA by HPLC-MS/MRM and HPLC-MS/MRM ${ }^{3}$ does not provide compelling arguments in favor of HPLC-MS/MRM ${ }^{3}$ particularly due to tradeoff in absolute signal sensitivity. However, following the examination of the extracted ion chromatograms provided by both approaches (Fig. 2), the added selectivity benefits of HPLC-MS/MRM ${ }^{3}$ become apparent. To allow comparison of samples by simultaneous or sequential detection, analytical methods for the analysis of neuropeptides need to be quantitative. To achieve this objective, the analytical strategy used must minimize the data measurement errors that might decrease the precision of each measurement and thus improve the sensitivity of the assay to detect concentration differences between samples. HPLC$\mathrm{MS} / \mathrm{MRM}^{3}$ methods may indeed improve the assay sensitivity by reducing significantly the potential of peak interferences and therefore reduce instrumental errors. Another benefit of HPLC-MS/MRM ${ }^{3}$ methods could be the possibility to improve the speed of HPLC separation and cycle time without having to deal with interferences observed in HPLCMS/MRM.

\section{Acknowledgements}

This work was funded by the National Sciences and Engineering Research Council of Canada (F. Beaudry NSERC Discovery Grant No. 386637-2010). F. Pailleux received a scholarship from the Région Rhône-Alpes in France (CMIRA Explora'doc 2013). 


\section{References}

1- CE Ferland, F Pailleux, P Vachon, F Beaudry. Neuropeptides. 45(2011):423-429.

2- CE Ferland CE, F Beaudry F, P Vachon. Phytother Res. 26(2012):1278-1285.

3- M. Rauh. J. Chromatogr. B. 883-884(2012) 59-67

4- T. Fortin, A. Salvador, J. P. Charrier, C. Lenz, F. Bettsworth, X. Lacoux, G. ChoquetKastylevsky and J. Lemoine Anal. Chem. 81(2009): 9343-9352

5- JA Trafton, C Abbadie, and AI Basbaum .J. Neurosci., 21(2001):3656-3664

6- R Patacchini, CA Maggi CA. Arch Int Phamacodyn Ther. 329(1995):161-184

7- WJ Martin, Y Cao, AI Basbaum. J Neurophysiol.91(2004): 1945-1954

8- VS Seybold. Handb Exp Pharmacol 194 (2009):451-491

9- F Beaudry. Anal Biochem. 407(2010):290-292 
Table 1. Selected peptide ions for the analysis in LC-MS/MRM and LC-MS/MRM ${ }^{3}$ modes.

\begin{tabular}{|c|c|c|c|}
\hline Peptide & Precursor ions & $\mathbf{M S}^{2}$ ions & MS $^{3}$ Ions \\
\hline \multirow{3}{*}{ SP } & \multirow{3}{*}{$674.7(\mathrm{z}=2)$} & 254.1 & 1086.7 \\
\hline & & $600.3^{1}$ & 1171.7 \\
\hline & & & 1199.8 \\
\hline \multirow{3}{*}{$d_{5}-S P$} & \multirow{3}{*}{$677.2(\mathrm{z}=2)$} & 254.1 & 1088.6 \\
\hline & & $602.8^{1}$ & 1176.8 \\
\hline & & & 1204.7 \\
\hline \multirow{3}{*}{ NKA } & \multirow{3}{*}{$567.3(\mathrm{z}=2)$} & \multirow{3}{*}{$\begin{array}{c}\mathbf{8 7 2 . 4}^{\mathbf{1}} \\
985.6\end{array}$} & 569.2 \\
\hline & & & 716.3 \\
\hline & & & 815.4 \\
\hline \multirow{3}{*}{$\mathrm{d}_{5}$-NKA } & \multirow{3}{*}{$569.7(\mathrm{z}=2)$} & \multirow{3}{*}{$\begin{array}{c}\mathbf{8 7 4 . 5}^{1} \\
990.5\end{array}$} & 569.2 \\
\hline & & & 716.3 \\
\hline & & & 815.4 \\
\hline
\end{tabular}

${ }^{1}$ Ions selected and isolated in the LIT to produce secondary product ions used to reconstruct LC$\mathrm{MS} / \mathrm{MRM}^{3}$ ion chromatograms 
Table 2. Summary of peptide quantification figure of merits obtained in LC-MS/MRM and LC$\mathrm{MS} / \mathrm{MRM}^{3}$ using fortified rat spinal cord samples.

\begin{tabular}{|c|c|c|c|c|c|}
\hline Peptide & & $\begin{array}{l}\text { Concentration } \\
(\mathrm{pg} / \mathrm{mg})\end{array}$ & 50 & 100 & 250 \\
\hline \multirow[t]{2}{*}{ SP } & MRM & $\begin{array}{l}\text { Mean } \\
\% \mathrm{Nom}^{\mathrm{a}} \\
\% \mathrm{CV}^{\mathrm{b}}\end{array}$ & $\begin{array}{l}48.1 \\
96.3 \% \\
24.1 \%\end{array}$ & $\begin{array}{l}98.7 \\
98.7 \% \\
7.4 \%\end{array}$ & $\begin{array}{l}250.3 \\
100.1 \% \\
6.1 \%\end{array}$ \\
\hline & $\mathrm{MRM}^{3}$ & $\begin{array}{l}\text { Mean } \\
\% \text { Nom } \\
\% \mathrm{CV}\end{array}$ & $\begin{array}{l}50.3 \\
100.6 \% \\
13.1 \% \\
\end{array}$ & $\begin{array}{l}98.2 \\
98.2 \% \\
4.7 \%\end{array}$ & $\begin{array}{l}253.1 \\
101.2 \% \\
7.2 \%\end{array}$ \\
\hline \multirow[t]{2}{*}{ NKA } & MRM & $\begin{array}{l}\text { Mean } \\
\% \text { Nom } \\
\% \mathrm{CV}\end{array}$ & $\begin{array}{l}49.1 \\
98.3 \% \\
14.7 \%\end{array}$ & $\begin{array}{l}109.8 \\
109.8 \% \\
3.5 \%\end{array}$ & $\begin{array}{l}228.3 \\
91.3 \% \\
4.3 \%\end{array}$ \\
\hline & $\mathrm{MRM}^{3}$ & $\begin{array}{l}\text { Mean } \\
\% \text { Nom } \\
\% \mathrm{CV}\end{array}$ & $\begin{array}{l}49.5 \\
99.0 \% \\
7.7 \%\end{array}$ & $\begin{array}{l}105.0 \\
105.0 \% \\
6.3 \%\end{array}$ & $\begin{array}{l}239.3 \\
95.7 \% \\
4.3 \%\end{array}$ \\
\hline
\end{tabular}

\footnotetext{
${ }^{\mathrm{a}}$ Accuracy

${ }^{\mathrm{b}}$ Precision
} 
Figure 1. Positive mode ESI MS ${ }^{\mathrm{n}}$ spectra of SP and NKA. (A) MS spectrum of SP showing a base peak at $[\mathrm{M}+2 \mathrm{H}]^{2+}$. (B) MS spectrum of NKA showing a base peak at $[\mathrm{M}+2 \mathrm{H}]^{2+}$. (C) $\mathrm{MS}^{2}$ spectrum of SP precursor $\mathrm{m} / \mathrm{z}$ 675. (D) $\mathrm{MS}^{2}$ spectrum of $\mathrm{NKA}$ precursor $\mathrm{m} / \mathrm{z} 567$. (E) $\mathrm{MS}^{3}$ spectrum of SP precursor m/z 600. (F) $\mathrm{MS}^{3}$ spectrum of NKA precursor m/z 872 .

Figure 2. Reconstructed ion chromatograms for transitions monitoring doubly charged species for SP and NKA in MRM and MRM $^{3}$ mode. (A) HPLC-MS/MRM extracted ion chromatogram of transition $675 \rightarrow[600+254]$ of reference standard (red line) and extracted spinal cord sample (black line) are compared with extracted spinal cord sample (blue line) HPLC-MS/MRM ${ }^{3}$ extracted ion chromatogram of transition $675 \rightarrow 600 \rightarrow[1087+1172+1200]$ for SP. (B) HPLCMS/MRM extracted ion chromatogram of transition $567 \rightarrow[872+986]$ of reference standard (red line) and extracted spinal cord sample (black line) are compared with extracted spinal cord sample (blue line) HPLC-MS/MRM ${ }^{3}$ extracted ion chromatogram of transition $567 \rightarrow 872 \rightarrow$ $[815+716+569]$ for NKA. 
Figure 1.

A

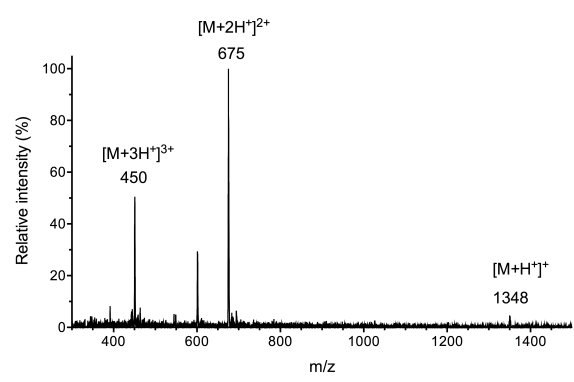

C



E

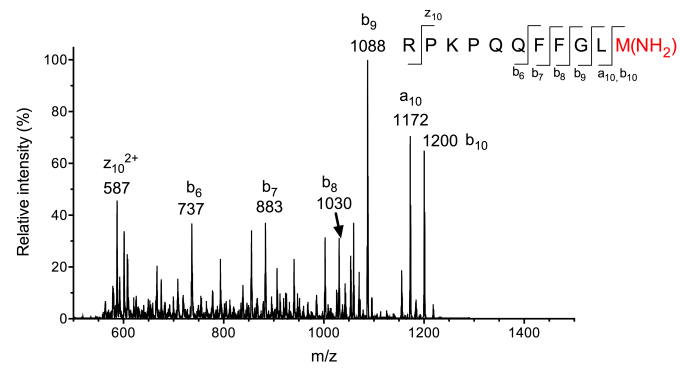

B

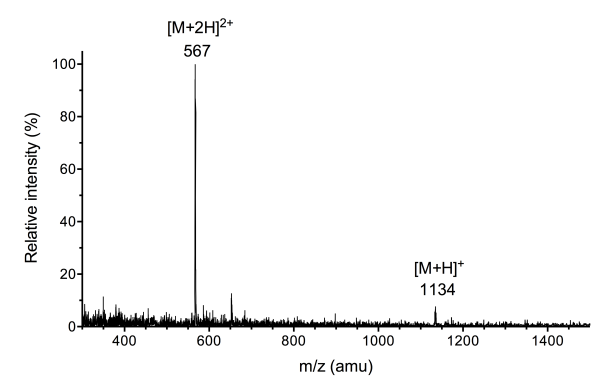

D

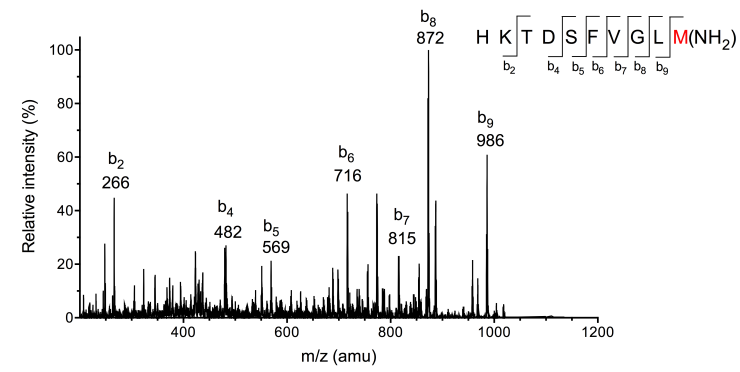

F

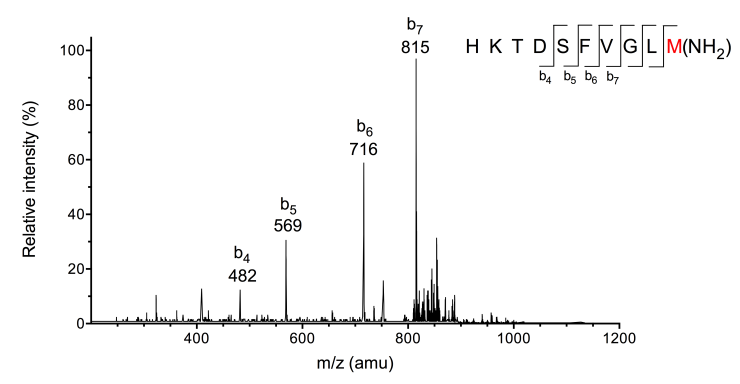


Figure 2.

A
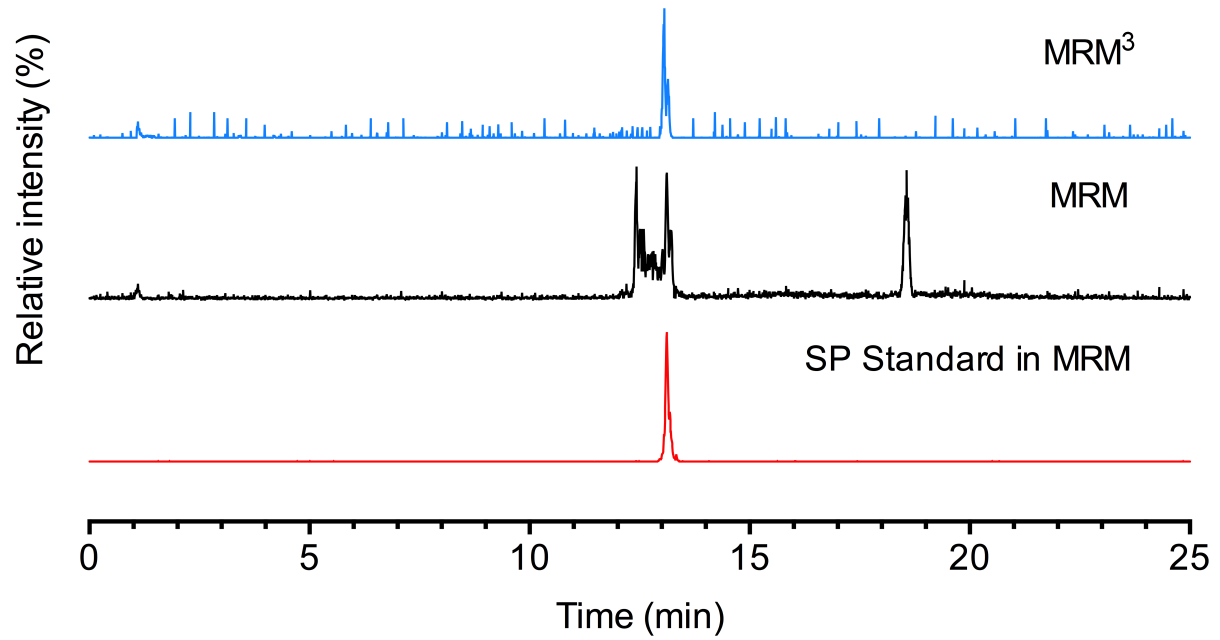

B

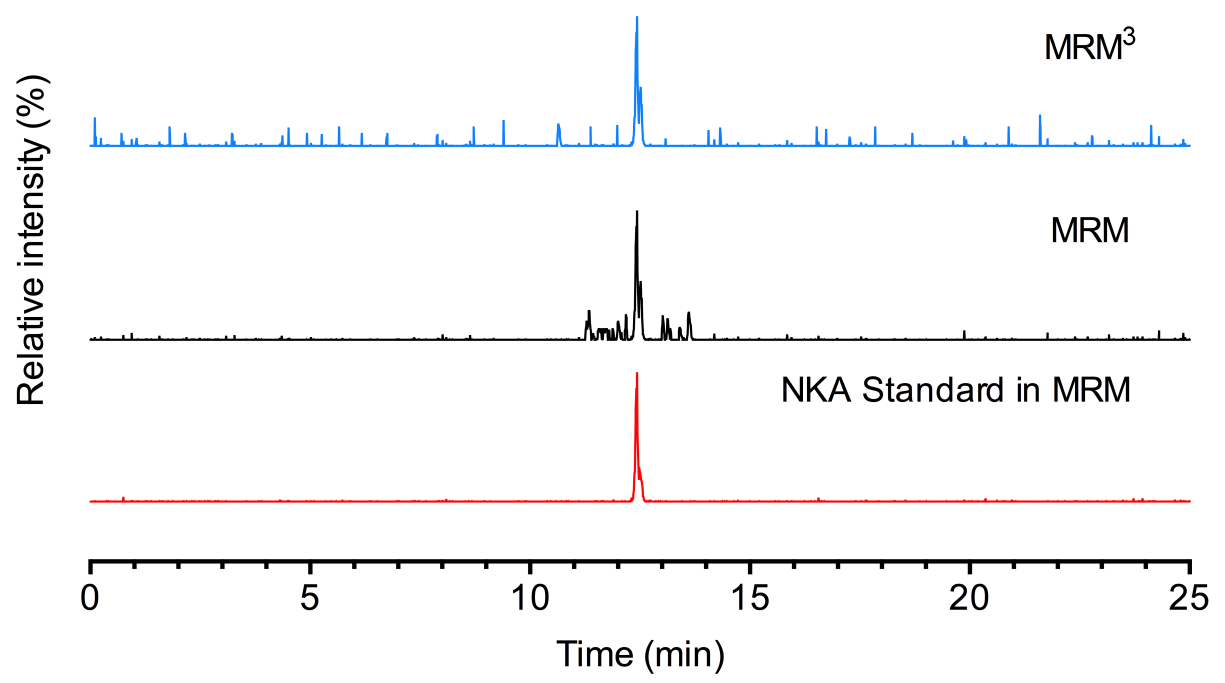

